\title{
Analytical Investigation of Channel Capacity of UWB-MIMO Systems
}

\section{Tai-Jung Huang}

Institute of Computer and Communication Engineering, Department of Electrical Engineering, National Cheng Kung University, Taiwan

Email: q38991182@mail.ncku.edu.tw

How to cite this paper: Huang, T.-J. (2018) Analytical Investigation of Channel Capacity of UWB-MIMO Systems. Int. J. Communications, Network and System Sciences, 11, 1-8.

https://doi.org/10.4236/ijcns.2018.111001

Received: December 16, 2017

Accepted: January 15, 2018

Published: January 18, 2018

Copyright $\odot 2018$ by author and Scientific Research Publishing Inc. This work is licensed under the Creative Commons Attribution International License (CC BY 4.0).

http://creativecommons.org/licenses/by/4.0/

(c) (i) Open Access

\begin{abstract}
Ultra-wide-band (UWB) technology combined with multiple input and multiple output (MIMO) provides a viable solution for achieving high data transmission rates of more than $1 \mathrm{~Gb} / \mathrm{s}$ in wireless communications. UWB is typically applied to short-range indoor environments and is therefore characterized by dense multipath propagation. In this type of environment, MIMO systems allow for a substantial improvement in the spectral efficiency by exploiting the inherent array gain and spatial multiplexing gain of UWB systems. This paper investigates the channel capacity of UWB-MIMO wireless technology and shows that UWB and MIMO designs improve the spectral efficiency logarithmically and linearly, respectively.
\end{abstract}

\section{Keywords}

Ultra-Wide-Band Communication, MIMO, Channel Capacity, Space-Time Coding, Beamforming

\section{Introduction}

UWB technology is typically applied to short-range indoor environments and is therefore characterized by dense multipath propagation. In such environments, MIMO systems allow for a substantial improvement in the spectral efficiency by exploiting the inherent array gain and spatial multiplexing gain of UWB systems. However, to maximize the performance of UWB-MIMO systems, further analyses of the UWB-MIMO channel capacity are required.

The capacity of narrowband MIMO fading channels has been the subject of both analytical [1] [2] and experimental investigation [3] [4]. However, the capacity of frequency-selective MIMO fading channels has attracted relatively little attention [4] [5] [6] [7]. The channel capacity of UWB-MIMO systems was ex- 
amined in [7] [8] [9] [10] [11], while that of UWB-SISO systems was investigated in [12]-[18]. Moreover, the authors in [12]-[18] also examined the channel capacity of $M$-ary PPM UWB systems. However, the UWB channel fading property was not taken considered. In [14] [16], the authors analyzed the channel capacity for multiple-access schemes based on time hopping and block waveform encoded $M$-ary PPM. However, while free-space propagation conditions were assumed, channel fading was once again ignored.

According to Edholm's law [19], indoor data rates of several Gbit/s are likely to become reality by around 2015. Thus, even though UWB systems offer enormous bandwidth, this bandwidth is likely to be insufficient. However, if the channel capacity of UWB systems can indeed be scaled in proportion to the number of transmit/receive antennas, this deficiency can be resolved by integrating UWB with MIMO systems. Nonetheless, the trade-off between the antenna and frontend amplifier design remains a significant challenge in UWB systems.

In general, only a minor loss in the frequency-selective channel capacity occurs in UWB systems when the signal-to-noise power ratio (SNR) is high (i.e., greater than $20 \mathrm{~dB}$ ) [20].

\section{System Model}

In analyzing the channel capacity problem, the following simplifying assumptions are made regarding the channel model:

Assumption 1: The noise $\mathbf{N}$ is zero-mean Gaussian with a power spectrum density (PSD) of $N_{0} \mathbf{I}_{N_{R}}$.

Assumption 2: The power of the transmitted signal is bounded by $\bar{S}$, i.e.,

$$
E\left[\mathbf{X}^{\mathrm{T}}(t) \mathbf{X}(t)\right]=\int_{-\frac{B}{2}}^{\frac{B}{2}} \operatorname{tr}\left(\mathbf{S}_{\mathbf{X}}(f)\right) \mathrm{d} f \leq \bar{S} .
$$

In the MIMO case, the input-output relation can be described by the following equation:

$$
\mathbf{Y}(t)=\sum_{l=1}^{L} \mathbf{A}_{l} \mathbf{X}[t-(l-1) \Delta \tau]+\mathbf{N}(t),
$$

where $\mathbf{X}(t)$ and $\mathbf{Y}(t)$ are $N_{T}$ and $N_{R}$-dimensional vectors of the transmit and receive signals, respectively, with $N_{T}$ and $N_{R}$ being the numbers of transmit and receive antennas. In addition, $\mathbf{A}_{l}, l=1, \cdots, L$ are amplitude fading matrices and $\mathbf{N}(t)$ is the receiver noise vector.

\section{Channel Capacity with Unknown CSI at Transmitter: UWB Case}

For an $N_{x}$-dimensional vector $\mathbf{x}$ with a Gaussian distribution, the differential entropy is given by [21]

$$
H(\mathbf{x})=\frac{1}{2} \log \left[(2 \pi e)^{N_{x}} \operatorname{det}\left(\mathbf{R}_{\mathbf{x}}\right)\right],
$$

where $\mathbf{R}_{\mathbf{X}}$ is the correlation matrix of $\mathbf{x}$. Introducing the term 


$$
\Phi(t)=\sum_{l=1}^{L} \mathbf{A}_{l} \mathbf{X}[t-(l-1) \Delta \tau]
$$

Equation (2) can be rewritten as

$$
\mathbf{Y}(t)=\Phi(t)+\mathbf{N}(t) .
$$

To address the capacity problem from an outage probability viewpoint, consider a given realization of $\bar{F}=\left(\mathbf{A}_{1}, \cdots, \mathbf{A}_{L}\right)$. Let the whole channel be divided into an infinitely large number of sub-channels in the frequency domain. Furthermore, assume that the sub-channel frequency range extends from $f$ to $f+\Delta f$. In each sub-channel, the spectrum of $\Phi(t)$ can be considered as flat with a value approximated by $\mathbf{S}_{\Phi}(f)$. Hence, the covariance matrices of $\Phi(t)$ and $\mathbf{N}(t)$ confined for sub-channel $[f, f+\Delta f]$ are given by $\mathbf{S}_{\Phi}(f) \Delta f$ and $N_{0} \Delta f \mathbf{I}_{N_{R}}$, respectively. Therefore, the mutual information $\Delta I \backslash_{\bar{F}}$ conveyed by this sub-channel, conditioned upon $\bar{F}$, is given by

$$
\begin{aligned}
\Delta I \backslash_{\bar{F}}= & \Delta f \log \left[(2 \pi e)^{N_{R}} \operatorname{det}\left(N_{0} \Delta f \mathbf{I}_{\mathbf{N}_{\mathbf{R}}}+\mathbf{S}_{\mathbf{\Phi}}(f) \Delta f\right)\right] \\
& -\Delta f \log \left[(2 \pi e)^{N_{R}} \operatorname{det}\left(N_{0} \Delta f \mathbf{I}_{\mathbf{N}_{\mathbf{R}}}\right)\right] \\
= & \Delta f \log \operatorname{det}\left[\mathbf{I}_{N_{R}}+\frac{1}{N_{0}} \mathbf{S}_{\boldsymbol{\Phi}}(f)\right]
\end{aligned}
$$

Note that in the argument above, the transmitted signal is assumed to have a Gaussian distribution when determining the capacity of the AWGN channel. Thus, taking the limit $\Delta f \rightarrow \mathrm{d} f$ in Equation (6), the conditional mutual information, denoted as $\Delta I \backslash_{\bar{F}}$, between $\mathbf{X}$ and $\mathbf{Y}$ is obtained as

$$
I \backslash_{\bar{F}}=\int_{-B / 2}^{B / 2} \log \operatorname{det}\left[\mathbf{I}_{N_{R}}+\frac{1}{N_{0}} \mathbf{S}_{\Phi}(f)\right] \mathrm{d} f .
$$

The conditional channel capacity $C /_{\bar{F}}$ is given by the maximization of $I \backslash_{\bar{F}}$ subject to the power constraint given in Equation (1), i.e.,

$$
C \backslash_{\bar{F}}=\max _{\int_{-B / 2}^{B / 2} \operatorname{tr}\left(S_{X}(f)\right) d f \leq \bar{S}} \int_{-B / 2}^{B / 2} \log \operatorname{det}\left[\mathbf{I}_{N_{R}}+\frac{1}{N_{0}} \mathbf{S}_{\Phi}(f)\right] \mathrm{d} f .
$$

It is noted that the derivation stated in Equation (7) is based on intuition. However, its rigorous proof can be obtained by repeating the same argument as that used in [22] based on the Karhuene-Loeve expansion. (Note, however, that the distribution of $F$ discussed in the present analysis is different from that in [22].)

Define

$$
\mathbf{H}(f)=\sum_{l=1}^{L} \mathbf{A}_{l} \mathrm{e}^{-j 2 \pi f(l-1) \Delta \tau} .
$$

Thus, $\mathbf{S}_{\boldsymbol{\Phi}}(f)$ can be expressed as

$$
\mathbf{S}_{\boldsymbol{\Phi}}(f)=\mathbf{H}(f) \mathbf{S}_{\mathbf{x}}(f) \mathbf{H}^{H}(f) .
$$

Substituting Equation (10) into Equation (8) yields 


$$
C \backslash_{\bar{F}}=\max _{\text {subject to (2.1) }} \int_{-B / 2}^{B / 2} \log \operatorname{det}\left[\mathbf{I}_{N_{R}}+\frac{1}{N_{0}} \mathbf{H}(f) \mathbf{S}_{\mathbf{X}}(f) \mathbf{H}^{H}(f)\right] \mathrm{d} f \text {. }
$$

It is clear that the maximum capacity is obtained when the equality for the power constraint in Equation (1) holds.

Now consider the optimal power design for the transmitted signal. The transmitter has no information regarding either $\mathbf{H}(f)$ or the noise $\mathbf{N}(t)$. Thus, in distributing the power in the transmitter, an intuitive approach is simply to allocate the power equally among all of the antennas and to uniformly distribute the power over the frequency band $[-B / 2,+B / 2]$. In other words,

$$
\mathbf{S}_{\mathbf{X}}(f)=\left\{\begin{array}{lc}
\frac{\bar{S}}{N_{T} B} \mathbf{I}_{N_{T}} & \text { when } f \in\left[-\frac{B}{2}, \frac{B}{2}\right] \\
0 & \text { otherwise }
\end{array}\right.
$$

Substituting Equation (12) into Equation (11), and using the substitution $u=2 \pi f \Delta \tau$ in the integral term and the fact that $1 / B=\Delta \tau$, it can be shown that

$$
\begin{aligned}
C \backslash_{\bar{F}} & =\int_{-B / 2}^{B / 2} \log \operatorname{det}\left[\mathbf{I}_{N_{R}}+\frac{1}{N_{0}} \frac{\bar{S}}{N_{T} B} \mathbf{H}(f) \mathbf{H}^{H}(f)\right] \mathrm{d} f \\
& =\frac{B}{\pi} \int_{0}^{\pi} \log \operatorname{det}\left[\mathbf{I}_{N_{R}}+\frac{\rho}{N_{T}} \tilde{\mathbf{H}}(u) \tilde{\mathbf{H}}^{H}(u)\right] \mathrm{d} u,
\end{aligned}
$$

where

$$
\rho=\frac{\bar{S}}{B N_{0}}
$$

and

$$
\tilde{\mathbf{H}}(u)=\sum_{l=1}^{L} \mathbf{A}_{l} \mathrm{e}^{-j(l-1) u} .
$$

\section{Channel Capacity with Known CSI at Transmitter: UWB-MIMO Case}

For the UWB-MIMO case, defining $\tilde{\mathbf{S}}_{\mathbf{X}}(u)$ as $\tilde{\mathbf{S}}_{\mathbf{X}}(u)=\mathbf{S}_{\mathbf{X}}(u B / 2 \pi)$, the conditional mutual information between $\mathbf{X}$ and $\mathbf{Y}$ given in Equation (7) can be re-expressed as

$$
I \backslash_{\bar{F}}=\frac{B}{\pi} \int_{0}^{\pi} \log \operatorname{det}\left\{\mathbf{I}+\frac{1}{N_{0}} \tilde{\mathbf{H}}(u) \tilde{\mathbf{S}}_{\mathbf{X}}(u) \tilde{\mathbf{H}}^{H}(u)\right\} \mathrm{d} u .
$$

In this case, the transmit power spectrum $\mathbf{S}_{\mathbf{X}}(f)$ (or equivalently $\tilde{\mathbf{S}}_{\mathbf{X}}(u)$ ) can be designed such that $C \backslash_{\bar{F}}$ is maximized for each realization of $\bar{F}$. The following derivations adopt the procedure outlined in [2], but adapt the original formulation to a UWB-MIMO channel. Since the matrix $\tilde{\mathbf{H}}^{H}(u) \tilde{\mathbf{H}}(u)$ is Hermitian, it can be diagonalized as

$$
\tilde{\mathbf{H}}^{H}(u) \tilde{\mathbf{H}}(u)=\mathbf{U}^{H}(u) \boldsymbol{\Lambda}(u) \mathbf{U}(u)
$$


and

$$
\breve{\mathbf{S}}_{\mathbf{X}}(u)=\mathbf{U}(u) \tilde{\mathbf{S}}_{\mathbf{X}}(u) \mathbf{U}^{H}(u),
$$

where $\mathbf{U}(u)$ is unitary and $\boldsymbol{\Lambda}(u)=\operatorname{diag}\left\{\lambda_{1}(u), \lambda_{2}(u), \cdots, \lambda_{N_{T}}(u)\right\} \ldots$ It is noted that $\mathbf{U}(u)$ and $\boldsymbol{\Lambda}(u)$ are both functions of the normalized frequency $u$. Using the matrix determinant identity $\operatorname{det}\left(\mathbf{I}+\mathbf{M}_{1} \mathbf{M}_{2}\right)=\operatorname{det}\left(\mathbf{I}+\mathbf{M}_{2} \mathbf{M}_{1}\right)$ for any two compatible matrices $\mathbf{M}_{1}$ and $\mathbf{M}_{2}$, it follows that

$$
\begin{aligned}
I \backslash_{\bar{F}} & =\frac{B}{\pi} \int_{0}^{\pi} \log \operatorname{det}\left\{\mathbf{I}+\frac{1}{N_{0}} \tilde{\mathbf{S}}_{\mathbf{X}}(u) \tilde{\mathbf{H}}^{H}(u) \tilde{\mathbf{H}}(u)\right\} \mathrm{d} u \\
& =\frac{B}{\pi} \int_{0}^{\pi} \log \operatorname{det}\left\{\mathbf{I}+\frac{1}{N_{0}} \mathbf{U}^{-1}(u) \breve{\mathbf{S}}_{\mathbf{X}}(u) \mathbf{U}(u)^{H^{-1}} \mathbf{U}^{H}(u) \boldsymbol{\Lambda}(u) \mathbf{U}(u)\right\} \mathrm{d} u \\
& =\frac{B}{\pi} \int_{0}^{\pi} \log \operatorname{det}\left\{\mathbf{I}+\frac{1}{N_{0}} \mathbf{\Lambda}^{1 / 2}(u) \breve{\mathbf{S}}_{\mathbf{X}}(u) \boldsymbol{\Lambda}^{1 / 2}(u)\right\} \mathrm{d} u .
\end{aligned}
$$

Note that the matrix $\Lambda^{1 / 2}(u) \breve{S}_{\mathbf{X}}(u) \Lambda^{1 / 2}(u)$ is non-negative definite. Thus, it follows that

$$
\operatorname{det}\left\{\mathbf{I}+\frac{1}{N_{0}} \boldsymbol{\Lambda}^{1 / 2} \breve{\mathbf{S}}_{\mathbf{X}}(u) \boldsymbol{\Lambda}^{1 / 2}(u)\right\} \leq \prod_{i=1}^{N_{T}}\left[1+\frac{1}{N_{0}} q_{i}(u) \lambda_{i}(u)\right],
$$

where $q_{i}(u), i=1, \cdots, N_{T}$, are the diagonal entries of $\breve{\mathbf{S}}_{\mathbf{X}}(u)$, and the equality holds when $\breve{\mathbf{S}}_{\mathbf{X}}(u)$ is diagonal. Therefore,

$$
I \backslash_{\bar{F}} \leq \frac{B}{\pi} \int_{0}^{\pi} \sum_{i=1}^{N_{T}} \log \left[1+\frac{1}{N_{0}} q_{i}(u) \lambda_{i}(u)\right] \mathrm{d} u .
$$

The following analysis determines $q_{i}(u)$ such that the integral term in the inequality in Equation (21) is further maximized under the constraint expressed in Equation (1). Let $\boldsymbol{\Theta}^{-1}$ be the Lagrange multiplier and construct the following function:

$$
\mathbf{J}\left(\mathbf{S}_{\mathbf{X}}\right)=\sum_{i=1}^{N_{T}} \log \left[1+\frac{1}{N_{0}} q_{i}(u) \lambda_{i}(u)\right]-\Theta^{-1} \sum_{i=1}^{N_{T}} q_{i}(u),
$$

based on the fact that $\operatorname{tr}\left(\mathbf{S}_{\mathbf{X}}(f)\right)=\operatorname{tr}\left(\breve{\mathbf{S}}_{\mathbf{X}}(u)\right)$. Notice that $\boldsymbol{\Theta}$ is a constant with a value independent of the frequency $u$. By setting $\partial \mathbf{J}\left(\breve{\mathbf{S}}_{\mathbf{X}}\right) / \partial q_{i}=0$, it can be seen that the optimal solution for $q_{i}(u)$ (i.e., the solution which maximizes $I \backslash_{\bar{F}}$ ) is given by

$$
q_{i}(u)=\left[\Theta-\frac{N_{0}}{\lambda_{i}(u)}\right]_{+} \text {. }
$$

By definition, set $q_{j}(u)=0$ if some eigenvalue of $\tilde{\mathbf{H}}^{H}(u) \tilde{\mathbf{H}}(u)$, say $\lambda_{j}(u)$, is equal to zero. Furthermore, let the constant $\boldsymbol{\Theta}$ be determined by the power constraint given in Equation (1) as

$$
\int_{-\frac{B}{2}}^{\frac{B}{2}} \operatorname{tr}\left(\mathbf{S}_{\mathbf{X}}(f)\right) \mathrm{d} f=\frac{B}{\pi} \int_{0}^{\pi} \operatorname{tr}\left(\breve{\mathbf{S}}_{\mathbf{X}}(u)\right) \mathrm{d} u=\frac{B}{\pi} \int_{0}^{\pi} \sum_{i=1}^{N_{T}}\left[\boldsymbol{\Theta}-\frac{N_{0}}{\lambda_{i}(u)}\right]_{+} \mathrm{d} u=\bar{S} .
$$

The conditional channel capacity is then given by 


$$
\begin{aligned}
C \backslash_{\bar{F}} & =\frac{B}{\pi} \sum_{i=1}^{N_{T}} \int_{F_{i \Theta}} \log \left[1+\frac{q_{i}(u)}{N_{0}} \lambda_{i}(u)\right] \mathrm{d} u \\
& =\frac{B}{\pi} \sum_{i=1}^{N_{T}} \int_{F_{i \Theta}} \log \left[1+\frac{\lambda_{i}(u)}{N_{0}} \Theta-1\right] \mathrm{d} u \\
& =\frac{B}{\pi} \sum_{i=1}^{N_{T}} \int_{F_{i \Theta}} \log \left[\frac{\Theta}{N_{0}} \lambda_{i}(u)\right] \mathrm{d} u,
\end{aligned}
$$

where $F_{i \Theta}$ denotes the intervals of $f$ in which $N_{0} / \lambda_{i}(u) \leq \Theta, i=1, \cdots, N_{T}$.

Then,

$$
\begin{aligned}
E[C] & =E\left[E\left[C \backslash_{\bar{F}}\right]\right] \\
& =E\left[E\left[\frac{B}{\pi} \sum_{i=1}^{N_{T}} \int_{F_{i \Theta}} \log \left[\frac{\Theta}{N_{0}} \lambda_{i}(u)\right] \mathrm{d} u\right]\right] \\
& =E\left[\frac{B}{\pi} \sum_{i=1}^{N_{T}} \int_{F_{i \Theta}} \log \left[\frac{\Theta}{N_{0}} \lambda_{i}(u)\right] \mathrm{d} u\right] \\
& =\frac{B}{\pi} \sum_{i=1}^{N_{T}} \int_{F_{i \Theta}} \log \left[\frac{\Theta}{N_{0}} \lambda_{i}(u)\right] \mathrm{d} u=C . \\
\Rightarrow & C=\frac{B}{\pi} \sum_{i=1}^{N_{T}} \int_{F_{i \Theta}} \log \left[\frac{\Theta}{N_{0}} \lambda_{i}(u)\right] \mathrm{d} u
\end{aligned}
$$

\section{Conclusion}

This paper has performed an analytical investigation into the channel capacity of UWB channels. It is noted that the channel correlation issue has not been considered in any great depth in this paper. However, a future study will evaluate the channel capacity for the case where the correlation among the elements of the fading matrices is considered from the viewpoint of fading pdf matrices. In conducting such an investigation, a problem arises in that it is necessary to specify the distribution, rather than the correlation matrix, of the $N_{R} \times N_{T}$-dimensional random matrix for the UWB system. While random matrix theory for the case where each entry is Gaussian is well established, very few reports on random matrix theory for other kinds of multivariate distribution are available in the literature. Moreover, even if such a multivariate distribution were available, generating the associated random matrices using the conventional Monte Carlo method still represents a major challenge.

\section{References}

[1] Foschini, G.J. and Gans, M.J. (1998) On Limits of Wireless Communications in a Fading Environment when Using Multiple Antennas. Wireless Personal Communications, 6, 311-335.

[2] Telatar, E. (1999) Capacity of Multi-Antenna Gaussian Channels. European Transactions on Telecommunications, 10, 585-596.

[3] Gnas, M.J., Amitay, N., Yeh, Y.S., Xu, H., Damen, T.C., Valenzuela, R.A., Sizer, T., Storz, R., Taylor, D., MacDonald, W.M., Tran, C. and Adamiecki, A. (2002) Outdoor BLAST Measurement System at $2.44 \mathrm{GHz}$ : Calibration and Initial Results. 
IEEE Journal on Selected Areas in Communications, 20, 570-583. https://doi.org/10.1109/49.995516

[4] Molisch, A.F., Steinbauer, M., Toeltsch, M., Bonek, E. and Thoma, R.S. (2002) Capacity of MIMO Systems Based on Measured Wireless Channels. IEEE Journal on Selected Areas in Communications, 20, 561-569. https://doi.org/10.1109/49.995515

[5] Borgmann, M. and Bolcskei, H. (2005) On the Capacity of Noncoherent Wideband MIMO-OFDM Systems. Proceedings of IEEE International Symposium on Information Theory (ISIT), Adelaide, September 2005, 651-655.

https://doi.org/10.1109/ISIT.2005.1523416

[6] Bolcskei, H., Gesbert, D. and Paulraj, A.J. (2002) On the Capacity of OFDM-Based Spatial Multiplexing Systems. IEEE Transactions on Communications, 50, 225-234. https://doi.org/10.1109/26.983319

[7] Malik, W.Q. and Edwards, D.J. (2007) Measured MIMO Capacity and Diversity Gain with Spatial Polar Arrays in Ultrawideband Channels. IEEE Transactions on Communications, 55, 2361-2370. https://doi.org/10.1109/TCOMM.2007.910700

[8] Ray, S., Medard, M. and Zheng, L. (2004) On MIMO Capacity in the Ultra-Wideband Regime. 38 th Asilomar Conference on Signals, Systems, and Computers, 7-10 November 2004, 1516-1520. https://doi.org/10.1109/ACSSC.2004.1399408

[9] Zheng, F. and Kaiser, T. (2004) On the Evaluation of Channel Capacity of Multi-Antenna UWB Indoor wireless Systems. Proceedings of the 2004 IEEE International Symposium on Spread Spectrum Techniques and Applications, Sydney, 30 August-2 September 2004, 525-529. https://doi.org/10.1109/ISSSTA.2004.1371755

[10] Zheng, F. and Kaiser, T. (2006) Channel Capacity of MIMO UWB Indoor Wireless Systems. In: Benedetto, M.-G.D. and Kaiser, T., Eds., et al. UWB Communication Systems-A Comprehensive Overview, Hindawi Publishing Corporation, New York, 376-409.

[11] Zheng, F. and Kaiser, T. (2008) On the Evaluation of Channel Capacity of UWB Indoor Wireless Systems. IEEE Transactions on Signal Processing, 56, 6016-6113. https://doi.org/10.1109/TSP.2008.2005089

[12] Erseghe, T. (2005) Capacity of UWB Impulse Radio with Single-User Reception in Gaussian Noise and Dense Multipath. IEEE Transactions on Communications, 53, 1257-1262. https://doi.org/10.1109/TCOMM.2005.852817

[13] Li, W., Gulliver, A. and Zhang, H. (2005) Performance and Capacity of Ultra-Wideband Transmission with Biorthogonal Pulse Position Modulation over Multi-Path Fading Channels. 2005 IEEE International Conference on Ultra-WideBand, Zurich, 5-8 September 2006, 225-229.

[14] Ramirez-Mireles, F. (2001) Performance of Ultra-Wideband SSMA Using TimeHopping and $M$-ary PPM. IEEE Journal on Selected Areas in Communications, 19, 1186-1196. https://doi.org/10.1109/49.926374

[15] Ramirez-Mireles, F. (2005) On the Capacity of UWB over Multipath Channels. IEEE Communications Letters, 9, 523-525. https://doi.org/10.1109/LCOMM.2005.1437358

[16] Ramirez-Mireles, F. and Scholtz, R.A. (1998) Multiple-Access with Time Hopping and Block Waveform PPM Modulation. International Conference on Communications, Atlanta, 7-11 June 1998, 775-779. https://doi.org/10.1109/ICC.1998.685115

[17] Zhang, J., Kennedy, R.A. and Abhayapala, T.D. (2003) New Results on the Capacity of $M$-ary PPM Ultra-Wideband Systems. International Conference on Communications, Anchorage, 11-15 May 2003, 2867-2871. https://doi.org/10.1109/ICC.2003.1204547 
[18] Zhao, L. and Haimovich, A.M. (2002) The Capacity of an UWB Multiple-Access Communications Systems. International Conference on Communications, New York, 28 April-2 May 2002, 1964-1968.

[19] Cherry, S. (2004) Edholm's Law of Bandwidth. IEEE Spectrum, 41, 58-60. https://doi.org/10.1109/MSPEC.2004.1309810

[20] Czylwik (1996) Kanalkapazitat von Breitband-Funkkanalen. Kleinheuvacher Ber., 39, 297-310.

[21] Cover, T.M. and Thomas, J.A. (1991) Element of Information Theory. J. Wiley \& Sons, Inc., New York. https://doi.org/10.1002/0471200611

[22] Ozarow, L.H., Shamai, S. and Wyner, A.D. (1994) Information Theoretic Considerations for Cellular Mobile Radio. IEEE Transactions on Vehicular Technology, 43, 359-378. https://doi.org/10.1109/25.293655 